\title{
ARTículo
}

\section{Aproximación etnográfica al cambio climático, sistemas rituales y experiencias locales en los Andes}

\section{An ethnographic approach to climate change, ritual systems and local experiences in the Andes}

\author{
Luis Alberto Suárez Rojas \\ https://orcid.org/0000-0003-4771-2788 \\ Isuarezr@unmsm.edu.pe
}

\author{
Sabino Arroyo Aguilar \\ https://orcid.org/0000-0003-0719-9078 \\ sarroyoa@unmsm.edu.pe
}

Universidad Nacional Mayor de San Marcos

\section{RESUMEN}

En este artículo analizamos la literatura disponible sobre el papel de las montañas sagradas, el cambio climático y la antropología. Exponemos algunos ejercicios etnográficos sobre este proceso de alcance global con referencias al Perú y otras partes del mundo. A partir de este contexto, ofrecemos una aproximación etnográfica de la relación entre las montañas sagradas, el cambio climático y los sistemas rituales ligados a los apus en la región de Apurímac, con el propósito de acercarnos a la experiencia local del cambio climático. Los datos etnográficos fueron recogidos durante un trabajo de campo en el distrito de Anccohuayllo, provincia de Chincheros, Apurímac, realizado en el 2019.

Palabras claves: Ruralidad; cambio climático; religión andina; etnografía.

\section{ABSTRACT}

In this article we analyze the available literature on the role of sacred mountains, climate change and anthropology. We present some ethnographic exercises on this process of global scope with reference to Peru and other parts of the world. From this context, we offer an ethnographic approach to the relationship among sacred mountains, climate change and ritual systems linked to the apus in the Apurimac region, with the aim of approaching the local experience of climate change. The ethnographic data were collected during field work conducted in 2019 in the district of Anccohuayllo, province of Chincheros, Apurimac.

Keywords: Rurality; climate change; Andean religion; ethnography.

(C) Los autores. Este artículo es publicado por ISHRA, Revista del Instituto Seminario de Historia Rural Andina de la Facultad de Ciencias Sociales de la Universidad Nacional Mayor de San Marcos. Este es un artículo de acceso abierto, distribuido bajo los términos de la licencia Creative Commons Atribución 4.0 Internacional (CC BY 4.0) [https://creativecommons.org/licenses/by/4.0/deed.es] que permite el uso, distribución y reproducción en cualquier medio, siempre que la obra original sea debidamente citada de su fuente original. 


\section{Introducción}

Hoy el cambio climático es parte de un proceso imparable que está mereciendo la atención tanto de políticos, jefes de Estado como de investigadores en muchas partes del mundo. El tema está en el centro de la agenda de las políticas públicas, y la antropología no puede estar ajena a esta preocupación. Por ello, el presente artículo ofrece algunos resultados de un proyecto de investigación en el marco del grupo de investigación Montañas Sagradas. Por lo tanto, en este artículo queremos ofrecer una aproximación etnográfica de la relación entre las montañas sagradas, el cambio climático y los sistemas rituales, según el referente empírico de testimonios y los datos etnográficos recogidos durante el trabajo de campo realizado, en octubre del 2019, en el distrito de Anccohuayllo, provincia de Chincheros, Andahuaylas.

Desde el enfoque de la antropología, las montañas sagradas fueron abordadas como un fenómeno religioso, desde la arqueología como un paisaje sagrado, e incluso desde la ecología, como parte del sistema de vida, y de la filosofía, disciplina que por cierto ha enfatizado la ecosofía para reconceptualizar la relación humanos-naturaleza. Desde nuestra perspectiva, las montañas sagradas constituyen un referente social, cultural y territorial que concita preocupación en muchas partes del mundo. Hoy, en los Andes, estas montañas cumplen un papel esencial en la racionalidad económica, estructura étnica y las expresiones simbólicas de los pueblos andinos. A pesar de la evangelización colonial y del culto a los santos patronos católicos, estas montañas sagradas no han perdido su función como divinidades tutelares, encarnadas en la figura de los apus y, en su contraparte, de la pachamama ${ }^{1}$ de los Andes.

Desde la perspectiva de las familias, los apus constituyen un arquetipo tutelar que habita en las montañas andinas, configurando paisajes sagrados y territorios donde se reproduce la dialéctica de lo sagrado y lo profano, como sugeriría Mircea Eliade (Eliade, 1981). Las divinidades tutelares descansan como entidades al interior de las montañas, sacralizando el espacio y cargándolo de una densidad de símbolos y ritos que nos remiten tanto al pasado como al presente. De ahí que la relación entre tiempo y espacio se complejiza, incorporando una gama de elementos actuales, siempre en dialogo con las preocupaciones de las actuales familias andinas.

Desde tiempos inmemoriales, las montañas sagradas o apus estuvieron estrechamente ligadas a la naturaleza y a los ciclos de reproducción natural; sin embargo, en la actualidad, están ocurriendo procesos de mayor alcance que escapan a la voluntad de los campesinos y sus familias. Nos referimos al cambio climático. Este proceso hace referencia a la pérdida de glaciares y a los cambios en la temperatura y gases de invernadero que generan impactos muy serios en las economías domésticas de carácter agrario y pastoril. Esta situación estimula a las familias a desplegar diversas respuestas a través de la racionalidad ecológica andina.

Debemos señalar que a este proceso global de cambio climático se suman otros eventos críticos como el retraso de lluvias, los incendios forestales y las heladas, o presiones globales. A ello debemos sumar otros factores que son parte de la nueva ruralidad, como el extractivismo formal e informal que viene impactando sobre el territorio, abriendo un escenario de disputa y conflicto por los recursos naturales. Todo esto genera tensiones, fricciones, desplazamientos y rupturas en programas sociales y universos religiosos, así como también respuestas sociales que devienen en conflictos que ponen en jaque a la vida política de la nación y la seguridad social de las comunidades.

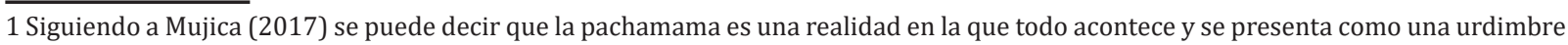
de relaciones. 


\section{Metodología}

En la primera parte del artículo hemos realizado una revisión de la literatura disponible sobre montañas sagradas y el cambio climático. Para ello, nos hemos apoyado en buscadores académicos y en la producción publicada en muchas latitudes del mundo; no obstante, nos hemos restringido a la producción antropológica. Por otro lado, este artículo es producto de un proyecto de investigación más amplio que recogió más datos etnográficos durante el 2019. En esa lógica, preparamos largos cuestionarios cualitativos, orientados a recoger la experiencia, percepción e implicaciones del cambio climático, desde la perspectiva de las familias campesinas de las zonas de Cusco y Apurímac. Además, se introdujo en estos cuestionarios todos los datos referentes al papel de las montañas sagradas, conocidas en el mundo andino como apus, para analizar y conocer si aún estaban activos en términos de culto y ritualidad. Como parte de este proyecto de investigación más amplio, incluimos observaciones y entrevistas a profundidad, no solo a campesinos sino también a técnicos, ingenieros y personal administrativo de los distritos visitados en las regiones de Cusco y Apurímac a finales del 2019. Finalmente, las observaciones, datos etnográficos y narrativas que presentamos fueron procesados durante el 2020.

\section{Revisión de la literatura}

\section{Las montañas sagradas y los sistemas rituales}

El estudio de las montañas sagradas ha merecido la atención de la arqueología, la antropología, la filosofía e incluso de algunas perspectivas teosóficas de finales del siglo XIX y comienzos del siglo XX. Además, hay abundantes referencias en textos sagrados de diversas latitudes. En suma, existen diversas reflexiones que apuntan a pensar que las montañas sagradas constituyen fenómenos sociales, culturales, territoriales y de biodiversidad, que concitan la preocupación en muchas partes del mundo. La referencia a montañas sagradas se puede encontrar en lugares tan diversos como Japón, Grecia, Medio Oriente, entre otras.

Desde la perspectiva de la antropología, podemos decir que usualmente son analizadas desde la lógica del fenómeno religioso: como totalidad o como experiencia fenomenológica. Desde la perspectiva de la arqueología es incorporada en la lógica del paisaje sagrado (Ceruti, 2013; Guzmán, 2019; Reinhard, 2012; Vitry, 2002, 2005). Otras perspectivas como la ecología sitúan al estudio de las montañas sagradas dentro del sistema de vida natural (Martínez, 2003). Así mismo, las humanidades, como la filosofía, han logrado estimular un enfoque interesante desde la ecosofía (Bugallo, 2014; Estermann, 2013; Maffesoli, 2017). En suma, existen muchos ángulos desde los cuales podemos abordar las montañas sagradas. Incluso, desde hace mucho tiempo, la psicología jungiana había versado sobre el papel de los lugares sagrados, los cuales fueron tomados como ejes del mundo simbólico y arquetípico, axis en términos cosmológicos.

De regreso, podemos sugerir que en el mundo andino, desde tiempos prehispánicos hasta el presente, tenemos múltiples representaciones de las montañas sagradas. En la perspectiva de las sociedades andinas, estas cumplieron un papel esencial en las interacciones económicas, en la estructura étnica y en las expresiones simbólicas de los pueblos andinos (Arroyo, 2006; Lecaros-Terry, 2001). Aún, con la evangelización colonial, las prácticas de extirpación de idolatrías y la introducción de los santos patronos (como fenómeno de asimilación), no han logrado desterrar ni olvidar a las montañas sagradas (Arroyo, 2012; Sánchez, 2006; Vitry, 2017).

Los apus como montañas sagradas y la pachamama son parte de las dinámicas rituales, las cuales se realizan a lo largo del ciclo agrícola-ganadero a través de oficiantes reconocidos por las comunidades. Estos toman algunas veces el nombre de altomisayuq, pongo, curioso, 
etc. (Brosseder, 2014; Ríos, 2004; Schlegelberger, 1996). Estas invocaciones están cargadas de una fe sólida, que ha resistido el paso de los años. Esto no excluye para nada el papel de los santos patronos y de la fe cristiana, que al parecer trabajan juntos para asegurar la fertilidad, como buena lluvia o la reproducción auspiciosa del ganado (Andía, 2003; Arroyo, 2012; Fuenzalida, 1979, 1980)

Desde la perspectiva de las familias, los apus constituyen un arquetipo de sacralidad, de fuerza y de energía protectora que habita en las montañas andinas y que se expande por la naturaleza y hace convergencia (tinkuy) con la pachamama, provocando la fertilidad y la expansión de la naturaleza y la realidad (Golte, 2003, 2004; Juárez, 2015; Millones, 2011). Ellos configuran un paisaje especial, un paisaje sagrado que nos demanda respeto y cariño como imperativos éticos (Ricard, 2007). Los apus mayores y menores se conectan, conversan y configuran entre sí relaciones de parentesco e interdependencia que forman una sacralidad pétrea, y a la vez activa, donde se reproduce la dialéctica de lo sagrado y lo profano.

En muchos de los casos, hemos recogido evidencia que apunta a sugerir que estas montañas sagradas fueron objeto de conversiones cristianas en la temprana colonia, y luego en la república. Incluso, existen algunos casos en los cuales frailes franciscanos fueron transfigurados y convertidos en apus o montañas sagradas de gran valor para las comunidades andinas (Arroyo, 2006). En otros casos, es posible que personajes notables de la vida de la colonia fueran absorbidos por las montañas sagradas, como el caso del Dr. Manuel Ayavi, en Uripa, donde la montaña además coexiste con su contraparte femenina.

Existen múltiples estudios, pero quisiéramos subrayar el trabajo de Xavier Ricard, quien sugiere para los pastores de la alta puna, que las montañas consideradas como apus cumplen el papel de pastores de hombres. Estos constituyen seres tutelares que cuidan a los hombres y mujeres, tal como lo harían ellos con los auquénidos. La relación entre los seres humanos y los apus es especial, íntima, y requiere la exigencia ética de la reciprocidad a través de las ofrendas que reproducen la compleja cosmovisión andina y su interpretación sobre el cuerpo, el tiempo y el espacio (Ricard, 2007).

En resumen, podemos decir que las montañas sagradas como objeto de reflexión e investigación nos remiten a un universo diverso de experiencias y de acercamientos, que en su conjunto apuntan a la existencia de una geografía sagrada, de una energía protectora y de una fuerza que vibra con ellos. Los apus viven en un otro-tiempo, pero entran y salen como si fueran dimensiones que se alternan, de la misma manera en que las narraciones míticas dan cuenta de seres humanos que entran y salen de un otro-tiempo. La reflexión sobre los apus y su contraparte, la pachamama, nos remite a un mundo donde todos los elementos son activos, dinámicos, expansivos. Los apus nos acercan a lo divino y a lo sagrado como algo inmanente. En último término, las divinidades tutelares descansan como entidades al interior de las montañas, sacralizando el espacio y cargándolo de una densidad de símbolos y ritos que nos remiten de manera dinámica tanto al pasado como al presente. Los ritos son el punto entre esa sacralidad inmanente con las preocupaciones de las actuales familias andinas.

\section{Cambio climático como objeto antropológico}

En la actualidad, el cambio climático constituye un problema que ha demandado políticas y compromisos internacionales. Así, los líderes han señalado que es un proceso irreversible que genera trastornos en los frágiles ecosistemas de montaña y alta montaña, y que provoca la pérdida de glaciares y los eventos extremos (como el friaje, por ejemplo). Las investigaciones aseguran que el cambio climático está provocando condiciones cada vez más secas a las comunidades de los 
Andes peruanos, provocando que los glaciares pierdan casi el 30\% de su área entre 2000 y 2016. En la 24 ${ }^{\text {va }}$ Conferencia de las Naciones Unidas sobre el Cambio Climático (COP24) en Katowice, (Polonia), se planteó que el cambio climático está generando efectos, tales como severas sequías y fenómenos climáticos extremos, además de poner en riesgo la seguridad alimentaria de las comunidades rurales. Desde nuestra perspectiva, como parte de una agenda futura, creemos que el acercamiento al cambio climático debería combinar una mirada multidimensional del binomio rural-urbano, una mirada comprensiva de la experiencia y visiones del cambio climático, y una reflexión de las implicaciones estructurales que esto genera en las sociedades de altura, en el mediano y largo plazo.

En esta sección, queremos presentar la revisión de algunos trabajos realizados entre los años 2010 y 2020, a fin de tener una perspectiva sobre lo avanzado y trabajado desde la antropología sobre el cambio climático. En ese sentido, destacamos el trabajo de Norgaard (2011), quien recuerda la relación entre variables ambientales y sociales como el cambio climático, el deterioro del medio ambiente y la migración. Su trabajo propone un conjunto de estrategias para la recolección de datos como la inferencia ecológica basada en las características del área, muestras individuales, series temporales, análisis multinivel, modelización basada en agentes y estudios cualitativos/etnográficos. Esta propuesta enfatiza la importancia de una estrategia integral para articular diversas entradas analíticas para comprender mejor la relación entre migración y medio ambiente.

Conceptos como adaptación y vulnerabilidad son centrales en la narrativa de los técnicos que instrumentalizan el cambio climático. En esa línea, existen dos investigaciones que desde la antropología exploran la adaptación, como Brugger y Crimmins (2013) y Sapkota et al. (2016). En esa dirección, el ejercicio analítico de Brugger y Crimmins (2013) sugiere que los políticos y técnicos del cambio climático han ejercido una notable influencia, colocando en el centro el concepto de adaptación, solo que en cierto sentido. Por esa razón, estos investigadores enfatizan la necesidad de ir más allá, llamando la atención sobre la importancia de la flexibilidad para pensar las estrategias de adaptación y no solo pensar los aspectos económicos y técnicos, además de no desvincularlas de debates como la desigualdad, la justicia y la transformación. Ellos exploran la manera cómo los residentes rurales del sudoeste de los Estados Unidos entienden, responden y planifican el tiempo y el clima en su vida cotidiana, y sugieren cómo esto podría ser parte de una estrategia más amplia de adaptación. Para comprender realmente la adaptación se debería considerar una aproximación ontológica que nos remita a la reciprocidad naturaleza-sociedad, al conocimiento situado y a leer el ajuste performativo de las actividades humanas a un entorno biofísico, social y de los valores.

Del mismo modo, Sapkota et al. (2016) subrayan que la vulnerabilidad al cambio climático es producto de la sociedad y sus configuraciones, en conexión con procesos sociales, culturales, económicos y políticos anclados en la densidad histórica. Exploran etnográficamente el concepto de vulnerabilidad en las comunidades rurales de la región de Panchkhal del distrito de Kavre, en las colinas medias de Nepal (2012 a 2015). Apoyándose en la teoría de Bourdieu sobre campo y práctica, analizan la vulnerabilidad, el aislamiento social, la autoridad financiera y la supremacía basada en el conocimiento que ejercen las elites comunitarias y los funcionarios públicos, identificando fuertes barreras sociales y jerarquías que influyen en la adaptabilidad a largo plazo de los grupos. No obstante, la capacidad de acción emerge bajo la figura de la movilidad humana, la acción colectiva y el cambio ocupacional.

Otra línea importante de análisis que conecta los estudios del cambio climático y la tradición antropológica es el análisis del impacto del cambio climático y su relación con las prácticas 
económicas y culturales de subsistencia. Desde esa perspectiva, destacamos los trabajos de Carothers et al. (2014), Marino (2015) y Graybill (2013). Así, a partir de una aproximación etnográfica, Carothers et al. (2014) documentan las observaciones locales del cambio climático y sus efectos en las prácticas de subsistencia en las comunidades rurales septentrionales del Ártico, y el papel que juega la manera cómo se conceptualizan el cambio climático y la pesca de subsistencia en tres comunidades iñupiat del noroeste de Alaska y seis comunidades athabascanas en la desembocadura del río Yukón. Carothers et al. (2014) sugieren que los cambios ambientales no se experimentan en el contexto de los cambios sociales que los acompañan, y que están continuamente reconfigurando las comunidades rurales de Alaska y las economías de subsistencia, tomando en consideración el medio ambiente y el cambio climático bajo la perspectiva holística del mundo humano-ambiental.

Por otro lado, el libro de Marino, Fierce climate, sacred ground: an ethnography of climate change in Shishmaref, Alaska (2015), aborda de manera muy convincente el hecho de que el cambio climático no es para nada un asunto teórico. Analiza etnográficamente la Isla Shishmaref en Alaska, hogar de los esquimales inupiaq, quienes viven de la cosecha de subsistencia, la cual se ve afectada por el cambio climático. Según Marino (2015), a pesar de que la isla Shishmaref está por desaparecer, es una comunidad unida y decidida a enfrentar esta dura realidad; por lo tanto, el estrés y la incertidumbre desafían la cultura y la identidad. Por otro lado, Marino (2015) enfatiza que las graves consecuencias del cambio climático a veces recaen sobre las comunidades que menos han contribuido con este dramático proceso. Esto nos remite a una dimensión política muy sugerente.

Como sabemos, el cambio climático provoca la pérdida de glaciares. Justamente, el trabajo de Graybill (2013) nos lleva a aproximarnos a lugares árticos y subárticos para analizar la vulnerabilidad al cambio climático, su impacto en ecosistemas, los medios de subsistencia y las formas de adaptación. Sin embargo, Graybill (2013) aborda las poblaciones del norte de Rusia, especialmente la región alpina subártica de Kamchatka, para acercarse etnográficamente a los aspectos emocionales y a las narraciones. En su perspectiva, esta sociedad considera que el clima cambia de forma natural y cíclica, y no considera que los humanos sean una fuerza lo suficientemente grande como para alterar los ciclos climáticos naturales, que los problemas medioambientales se puedan solucionar con la tecnología y que existe una falta de conocimiento sobre la ciencia del cambio climático. Así, al abordar las emociones, nos recuerda que puede conectarse con otros campos como la economía, la política y la cultura.

Por otro lado, desde una perspectiva crítica, Rudiak-Gould (2011) busca analizar la manera cómo el discurso científico global sobre el cambio climático se percibe desde la experiencia de las comunidades. Para ello, toma el caso de las Islas Marshall, demostrando la influencia de este discurso en las opiniones locales sobre el cambio ambiental, y asegura que debemos comprender la interacción de ambos discursos y ligarlo al acceder a las nociones locales (emic) del cambio climático.

Hasta la fecha, la antropología ha jugado un rol importante en el desarrollo de estudios para observar y registrar la percepción de la población sobre el calentamiento global, pero lo que propone es un camino complementario. En esa línea, debemos resaltar el trabajo de Roncoli et al. (2016), que busca apoyarse en la antropología para comprender la manera de percibir, experimentar y responder al entorno y a los sistemas de significados y relaciones sociales. Ahí, el trabajo de campo etnográfico contribuye a producir conocimientos situados sobre el clima y la cultura. Y aunque usualmente la antropología se acercó al pasado de los grupos humanos, estos investigadores consideran que el eje del cambio climático nos permitiría proyectarnos hacia el 
futuro. Desde su perspectiva, la antropología podría ser capaz de comprender el clima a partir del significado cultural y las prácticas sociales, que escapan de los estudios cuantitativos.

Segun Roncoli et al. (2016), existen cuatro axiomas que articulan diferentes formas del cómo, desde la cultura, nos aproximamos al cambio climático. Primero, cómo las personas perciben el cambio climático a través de lentes culturales (o percepción); segundo, cómo las personas comprenden lo que ven, basándose en sus modelos mentales y lugares sociales (o conocimiento); tercero, cómo valoran lo que saben en términos de significados compartidos (o valoración); y cuarto, cómo responden, individual y colectivamente, sobre la base de estos significados y valores (o respuesta). Por otro lado, enfatizan que el cambio climático está ligado a las fluctuaciones, interconexiones mundiales y desafíos para movernos en terrenos epistemológicos inexplorados. Asimismo, reconocen la naturaleza politizada de la ciencia del clima y su influencia en las decisiones políticas que afectan a las comunidades indígenas, los grupos marginales y los pobres.

De hecho, la antropología enfatiza el papel de los valores culturales y las relaciones políticas. En ese sentido, precisamos el trabajo de Barnes et al. (2013), quienes sugieren que la antropología puede considerar estas variables para analizar cómo entendemos y reaccionamos ante el clima, además de no soslayar el contexto histórico del debate sobre el clima. Por esa razón, la antropología contribuye con una visión holística sobre los sistemas humanos y naturales y el papel del cambio (cultural, social, político y económico). Finalmente, este trabajo busca ir más allá de las visiones locales del clima para comprenderlo desde la ciencia contemporánea y preguntarse cómo circula el conocimiento científico. En todos los casos, se requiere una dinámica de retroalimentación positiva de las ciencias, sobre las ciencias sociales y viceversa.

Así mismo, debemos destacar las contribuciones sobre el cambio climático y sus efectos en los sistemas culturales y la organización social en la arqueología (Balter, 2007; Kuper y Kropelin, 2006; Migowski et al., 2006; Richerson, 2001), la ecología histórica (Crumley 1994; McIntosh, 2000; Oldfield, 1993), y la ecología cultural (Bogin, 1982; De Menocal, 2001; Peterson y Haug, 2005).

\section{Etnografía y cambio climático en el Perú}

Al respecto de la literatura sobre cambio climático en el Perú, debemos destacar el trabajo de Paerregaard (2013), el cual toma en consideración los datos etnográficos actuales como aquellos recogidos en la década de los ochenta, y analiza la práctica ritual y la creencia religiosa de una comunidad rural de los Andes. Así también, explora cómo ha cambiado en los últimos 27 años y cómo se perciben estos cambios. Su trabajo sugiere que los campesinos rechazan la idea de que son las actividades humanas en otras partes del mundo las que causan problemas ambientales en su comunidad, y que los desafíos deben abordarse localmente. Sobre el medio ambiente, resalta que es pensado como co-habitado por humanos y no humanos, y además que el cambio climático ha causado un cambio en su visión del mundo. En esa línea, encontramos también la contribución de Cometti (2020), quien, con una estrategia etnográfica, da cuenta de la experiencia del cambio climático entre los Q'ero (Cusco), quienes poseen una visión del medio ambiente como co-habitado. Más recientemente, Paerregaard (2020) ha sugerido que la investigación sobre el cambio climático es una ciencia posnormal, y que por lo tanto debe incluir las diversas tradiciones y los métodos académicos para realmente escuchar las voces de quienes se ven impactados por el cambio climático.

En un análisis comparativo entre Perú e Italia, Jurt et al. (2015) desarrollan un ejercicio etnográfico tanto en Carhuaz, en la Cordillera Blanca (Perú), como en Stilfs, en los Alpes del Tirol del Sur (Italia), identificando las percepciones sobre el cambio climático en estos lugares 
caracterizados por la pérdida de glaciares. Asimismo, subrayan que es vital conocer y comprender las respuestas al cambio climático desde la experiencia, las visiones, preocupaciones locales, y entender el papel que juega su lugar en el mundo. Aunque es necesario adoptar medidas para mitigar el cambio climático, es clave comprender que no es algo compartido por todos, y ahí radica el desafío.

En el contexto del parque nacional de la papa, en Cusco, Walshe y Argumedo (2016) exploran etnográficamente comunidades rurales, analizando las capacidades de responder ante el desafío del cambio climático. Estas tienen relación con los valores culturales como el ayni, el ayllu, el yanantin y la chanincha. Lo importante es que su trabajo resalta que las estrategias de adaptación a veces soslayan estos valores e incluso los erosionan.

Por otro lado, las conexiones entre el discurso sobre el cambio climático y las percepciones locales fueron exploradas por Weber-Alvarez (2018) a través de un ejercicio etnográfico acerca de los discursos nacionales (Perú), regionales (Cusco) y locales (Chumbivilcas) sobre el cambio climático. Weber-Alvarez subraya la necesidad de comprender cómo se transmite, procesa y adapta el discurso sobre el cambio climático mundial en un contexto local.

Finalmente, el trabajo de Orlove et al. (2019) aborda etnográficamente tres comunidades impactadas por la pérdida de glaciares, apoyándose en una técnica mixta de investigación de entrevistas a profundidad, grupos de discusión, etc. Sugiere que las comunidades poseen una observación muy precisa sobre el cambio climático, pero es necesario movernos a un marco de acción comunitaria para emprender acciones. Además, sugiere que es posible dudar sobre el modelo percibir-predecir-actuar, tan característico del discurso sobre el cambio climático.

\section{Aproximación etnográfica}

\section{Contexto de la Región}

El proyecto de investigación, cuyos resultados compartimos en este artículo, nos llevó a recoger experiencias, observaciones y datos etnográficos en regiones de Cusco y Apurímac. Hemos podido evidenciar una fuerte preocupación por el alterado ritmo de las lluvias. Muchas familias sugieren que planificar las cosechas se está convirtiendo en un asunto difícil de manejar, y para ello se apoyan en sus fuerzas tutelares, tanto los apus y la pachamama, como también sus santos patronos, con la finalidad de asegurar una buena temporada. La ausencia de lluvias lleva a que muchas familias quemen los arbustos secos en la ladera de los cerros o en las zonas altas. Del mismo modo, la presencia de la lluvia loca, es decir, la lluvia abundante y prolongada, también afecta los sembríos y la calidad de la tierra. A esto se suma la presencia de plagas, insectos y los cambios de temperatura.

Desde la perspectiva de las familias campesinas, a veces demasiado calor afecta y estresa los sembríos; por otro lado, la presencia de granizada los coloca en una posición vulnerable, ya que para tratar de contrarrestar el granizo muchas familias utilizan cuetes para ahuyentarlo. Pero ocurre todo lo contrario con la helada, pues en este caso ellos no pueden controlar la caída de la temperatura, lo que en muchos casos afecta tanto a las familias como al rebaño. Esto es análogo a lo que sucede en otras regiones, por ejemplo, en Ayacucho y Huancavelica, donde atenúan el granizo con el humeado, que consiste en quemar chamizos (retama seca) al lado de las parcelas a merced de la helada ${ }^{2}$, a fin de que el humo ahuyente a la helada, que amenaza con quemar las plantas y sus frutos. Según las familias, en el campo sienten que ocupar las faldas de los cerros

2 De acuerdo con Vallejo y Alvarado (2015), las heladas son fenómenos atmosféricos que ocurren por la disminución de la nubosidad en los Andes. Se produce cuando la temperatura ambiental disminuye más allá de los cero grados centígrados, durante los meses de junio, julio y agosto. Este fenómeno se origina, en mayo medida, en las zonas ubicadas a más de 3500 metros sobre el nivel del mar. 
o de los apus es más seguro tanto la producción como la vida de sus ganados, a diferencia de las pampas o las planicies alejadas de los dioses tutelares.

En el presente, tanto hombres como mujeres del campo reconocen que el clima ha cambiado, que es difícil tener un control y hacer predicciones sobre él. Ellos aseguran que viven un tiempo muy difícil, de incertidumbre, de inestabilidad y de tensiones. En este contexto, la presencia de las fuerzas tutelares adquiere gran importancia para las familias. Tanto las semillas como los animales son bendecidos en nombre de los apus y la pachamama. Para ambos las familias se organizan para celebrar y ofrecer ricas y abundantes ofrendas.

De hecho, el tema de la ausencia de lluvias y largas temporadas secas provoca que muchas familias se organicen para llevar operaciones mágico-rituales a cargo de un altomisayoq, curiosos o pongos ${ }^{3}$ en las zonas altas, donde se encuentran lagunas reconocidas como poderosas. Estos operadores mágicos dominan la comunicación con los apus y las fuerzas arcanas de la naturaleza, pues conocen y saben cómo despertar el encanto de las lagunas y el poder de los apus. En ese sentido, en varios lugares donde encontramos lagunas importantes nos han hecho referencia a rituales para atraer la lluvia y alimentar el poder de las lagunas y de los cerros, como aseguran los pobladores de la comunidad de Anqobamba de Chaupimarka de Aymaraes, donde lanzar piedras ritualmente a la laguna de Supoqocha busca propiciar lluvias torrenciales, aunque es como castigo del Apu Suparaura ${ }^{4}$.

Esto permite evidenciar el papel importante de los apus en este contexto de cambio climático, pues constituye un referente, una guía y un apoyo. Y por esa razón, en algunos lugares donde hay presencia de minería informal o proyectos incipientes, la población ha reaccionado con firmeza para echar a los mineros, señalando que profanan un espacio altamente sagrado; véase como un ejemplo de esto, la defensa del Apu Pisti en Chalhuanca (Aymaraes).

En la siguiente sección, ofrecemos algunos datos etnográficos alrededor del Apu Ayavi y Apu Piti, a partir de los resultados de una entrevista en profundidad, seleccionada a propósito como ejemplo de esta visión y experiencia local sobre el cambio climático y los sistemas rituales.

\section{El distrito de Anccohuayllo y sus apus}

El distrito de Anccohuayllo pertenece a la provincia de Chincheros, y es la tercera provincia con mayor población después de Andahuaylas y Abancay. Antaño, el distrito de Anccohuayllo llevaba el nombre de Uripa. Los locales consideran que Uripa proviene del vocablo Europa, pues la calidad paisajística del valle atrajo a muchos españoles en la Colonia, quienes admiraron el clima y sus nevados. El distrito de Anccohuayllo posee una población de 10,388 habitantes y todos los centros poblados están por encima de los $3000 \mathrm{msnm}$. La gran mayoría se dedica a la agricultura y la ganadería. Destacan los cultivos de papa, maíz, trigo, olluco, mashua y quinua; además, encontramos cultivos secundarios como las frutas, las hortalizas y los frijoles. A estas actividades se suma el comercio, el cual constituye otra fuente de acceso a dinero. La ubicación de este distrito se ve beneficiada por su localización en el corredor Ayacucho-Apurimac-Cusco.

En este distrito encontramos vestigios de la cultura chanca y la cultura inca. De hecho, el nombre de Anccohuayllo corresponde a un antiguo jefe chanca que se enfrentó a los incas, razón por la cual la población reconoce que fue el último guerrero chanca. Así mismo, podemos visualizar

\footnotetext{
3 Según Polia (1996), en los Andes del Perú, los operadores mágicos llevan diversos nombres como: curanderos, chamanes, pongos, curioso, altomisayoq o pampamisayoq. El nombre varía según la región.

4 Reconocido como el apu principal en la región de Apurímac. Es una de las divinidades mayores de la provincia de Aymaraes, registrado por Guamán Poma como una de las «Wakas Mayores de Chinchay Qocha» (Guaman Poma, 1980, p. 187), tributado con mucho oro y sacrificios humanos.
} 
la presencia inca gracias al gran sistema vial conocido como Qhapaq Ñan (Gran Camino Inca) y que, según los campesinos y familias del distrito, continúan utilizando para desplazarse entre los diferentes centros poblados y comunidades.

De igual modo, se vienen produciendo fenómenos naturales que ejercen presión sobre las familias campesinas. Durante el trabajo de campo se registraron algunos cambios climatológicos; por ejemplo, en noviembre se registró en Uripa una fuerte granizada por más de media hora, dañando los cultivos de maíz y papa. En este contexto, los agricultores hicieron serias demandas al gobierno local con el fin de obtener su apoyo. Esto significa que las familias en el campo no son actores pasivos de estos procesos globales, ya que también están llamando la atención del gobierno local.

De acuerdo al trabajo de Luis Mujica (2017), se puede afirmar que Uripa se encuentra rodeada de cerros que configuran su paisaje natural y cultural, entre ellos: Ataqara, Tururumi, Hatunhuayccu, Marayniyoc, Potrero, Apu Ayavi (el más importante en la zona y muy mentado en otras), Qoya, Arpa, Huayratinqa, Callhuaysumuqa, Ccarhuaypata, Llampuyaquichca, Andas, Cruz pata, Rihuna, Ercuilla y Rocotomocco.

\section{Actividad y poder de los dioses del cerro: los rostros del Apu Doctor Manuel Ayavi y el Apu María Piti y Angas}

Tuvimos la oportunidad de conocer y sostener una conversación muy nutrida e importante con la señora Juana Cárdenas, de 68 años de edad. Ella nos recibió en su casa de Uripa, en medio del campo y muy cerca de la carretera. Hace algunos años su esposo falleció y hoy vive con sus hijos y nietos. La señora Juana no logró conocer a sus abuelos, no obstante, asegura que su padre fue de Pacaicasa (Ayacucho) y su madre de Andahuaylas. Ambos se quedaron allí por su negocio y compraron sus propiedades, y ella nació en Uripa.

Desde que ella era niña con "comprensión de las cosas», al apu del pueblo se le conocía por el nombre de Doctor Manuel Ayavi; aunque ella no sabe la razón de su nombre, piensa que este nombre proviene de mucho tiempo atrás. Ella nos relató algunas experiencias de vital importancia por la intimidad y cercanía con el Apu Manuel Ayavi. Su relato nos ayudará mucho a comprender esta dimensión compleja y relacional de los apus en el mundo mágico ancestral y simbólico en Uripa.

Hace muchos años, cuando ella tenía 12 años de edad, su hermano Néstor Cárdenas solía hacer diversos trabajos por muchas zonas de la sierra y de la selva. Pero, en una ocasión retornó enfermo de un viaje que hiciera a la selva. Ellos concluyeron rápidamente que la montaña (selva) lo dañó (enfermó). Por esa razón, sus padres, muy preocupados, tuvieron que salir en busca de ayuda. En principio, visitaron a médicos, pero no lograron paliar su estado crítico, y su situación seguía agravándose. Dado su estado de salud, algunas personas aconsejaron a sus padres que fueran a Ocobamba, pues ahí vivía un buen curandero que «llama y trata con el cerro (apu)». Aunque en la actualidad muchos declaran que los pongos o curanderos se están extinguiendo, otros afirman que aún podemos encontrar alguno en Quispimarka.

El nombre del curandero era Fabián Díaz y los recibió en Ocobamba. Rápidamente pudo examinar a su hermano Néstor y luego de un procedimiento mágico diagnosticó que el monte (árboles) lo había enfermado. La situación, según el curandero, fue que uno de los árboles que cortó tenía sangre. La señora Juana hace memoria y recuerda que, en aquellos años, su hermano estuvo en la selva de San Francisco de Ayacucho. En ese tiempo, los enganchadores venían y con- 
trataban a muchos trabajadores de esta región para trabajar en la selva, y es altamente probable que su hermano haya cortado un árbol sin permiso de los apus.

La señora Juana no recuerda si el diagnóstico fue a través de naipes u otras prácticas adivinatorias. Pero ese fue el diagnóstico del pongo (operador mágico), quien sugirió que para curarlo era necesario llevarle su pago al apu y luego cambiarle la suerte. Así, el curandero aceptó visitar la casa de sus padres dentro de cinco días para tratar a su hermano. Pasaron los días y llegó en la fecha señalada. Una vez que el curandero llegó a casa reunió a la familia y mandó a comprar platos, un par de pañuelos de mujer joven, entre otras cosas. Ella fue testigo del proceso curativo y nos explica que cuando estuvo rezando como parte del ritual de curación, llegó el Apu Doctor Manuel Ayavi y dos apus de la selva cuya identidad desconocía, aunque lo importante es que ellos entablaron una conversación. Luego, el curandero confirmó con mayor exactitud la causa de la enfermedad de su hermano: él no solo había llegado a tocar la sangre de uno de los árboles que cortó, sino que además habría tocado su corazón.

Durante el ritual se presentaron dos apus de la selva y conversaron con el Apu Manuel Ayavi, confirmando que ellos habían enfermado a su hermano porque él había cortado y maltratado su árbol. Para cambiar esto, ellos debían repararlos con un pago (o pagapu ${ }^{5}$ ), pues de lo contrario, no lo soltarían. En esta lógica ritual, el Apu Doctor Manuel Ayavi se define como abogado defensor y, a lo largo de la conversación, la señora Juana pudo percibir que ellos son como nosotros. Así el Apu Doctor Manuel Ayavi tenía la voz delgada, el otro tenía voz gruesa y el otro menos. No recuerda sus nombres por ser menor de edad, pero estuvo atenta al proceso de curación.

Luego, con una diversidad de elementos procedieron al pago/despacho (pagapu) a cargo del curandero. Así, en la mesa ritual colocaron platos, los cuales desaparecieron sin que ella supiera a dónde se los habían llevado. Había la flor clavel, gaseosa, vino, caramelo, coca, cigarro, a todo lo cual añadieron fruta y de ese modo toda la mesa se llenó. No sabe el paradero de esos platos, aunque sí es consciente que el curandero sí sabía dónde debía llevarlos. Tras la ejecución del ritual, recién los soltó y su hermano se restableció. Desde aquella vez, su hermano nunca más retorno a la selva y tuvo mucho miedo y respeto.

En este distrito encontramos varios apus locales; por ejemplo, María Piti Angas del barrio Challwani y de Tukumachay. Apu Pakutullo de Miraflores, Ataqara de Qollpapampa, Apu Raya-Pata es de Chuparo. Y para Chincheros es Teja Wasi, Chipikuy, Ataqara, que se ubica al frente del Apu Ayavi. En ese lugar estaría el manantial Llamachayuq y en la parte alta de la quebrada está Ataqara; y a la vuelta está el pueblo Ongoy, que baja por una carretera.

Al parecer el Apu Doctor Manuel Ayavi tiene su complemento femenino: el Apu María-Piti y Angas. También podemos encontrar el Apu Riwina, que es uno de los más altos y donde justamente encontramos una antena de telecomunicaciones, así como su correspondiente apu menor del mismo nombre, Riwina. Este apu es muy importante porque ahí se realizan los pagos para la producción agrícola. La señora Juana nos enseña que debemos decir así: «Apu Riwina, mamapacha, apu Riwina aquí te traigo tu bendición». Esta pequeña rogativa se realiza con la hoja de coca, con el propósito de asegurar una buena producción. También se vierte la chicha, nada más y no hay nada especial.

Como hemos señalado arriba, el Doctor Manuel Ayavi posee las cualidades de un abogado. Los padres de Juana aseguran que el Apu Ayavi estuvo en juicio por su nevado (riti), mientras nos señala la figura de llamita, imagen que se forma cuando cae la nieve en el cerro. Cuando

5 Un pagapu es un pago, que constituye una ofrenda ritual compuesta por diversos elementos. En los Andes, los pagos son considerados ofrendas a la pachamama o a los apus, y su composición varía según la región en los Andes. 
está en juicio, el Apu Ayavi se presenta en Lima como Doctor para hablar con los otros apus y, aunque ella no sabe cuáles son, asegura que siempre se reúnen en Lima. Aunque, los mayores dicen que siempre competían con el apu del pueblo de Ongoy. En términos generales, los apus se comunican, viajan y desarrollan litigios por sus propiedades en Lima.

Además, la señora Juana logró escuchar a los mayores que los apus tienen la capacidad de presentarse como un caballero montado en su caballo y en otras ocasiones como una vizcacha (Lagidium viscacia). En una ocasión, su padre contó que caminó con su hermana a la parte baja por la tuna y ahí ella se asustó. Luego acamparon en la ribera del río Pampas y por seguridad prepararon una fogata en la noche. Luego de la cena, cerca de las ocho de la noche, escuchó que de la parte alta del cerro caían piedrecillas, mientras que los mayores no lograron despertarse. Luego se habría escuchado dos voces que, al parecer, eran diálogos entre el cerro y el que estaba al frente. Luego, su hermana enfermó y después de un mes murió. Ellos creen que los médicos provocaron la confusión al diagnosticar que tenía paludismo, cuando en realidad el apu le había «ganado» (quitado) su espíritu. Esta es una cualidad peligrosa de los apus, pues son capaces de robar la sombra de uno. Ellos tienen su «qaqcho» (poder inmanente, fuerza de poseer).

Luego, la señora Juana nos narró que, en cierta ocasión, el vecino de su padre, quien era su compadre, lo había regañado por no haberlo llevado a tiempo al curandero. A pesar de todo, lograron visitar al curandero, quien lo examinó y con gran pesar se dirigió a ellos y no aceptó curarlo porque ya era demasiado tarde. Con tristeza tuvieron que regresar a casa. Más tarde, su padre salió de casa en una tarde de neblina, y en medio de la bruma y la falta de visibilidad logró observar que su compadre caminaba con dirección al cerro. Al poco tiempo falleció y la familia y los amigos creen que el apu se lo llevó. Además, durante la noche de su muerte, el apu habría llegado a su casa, haciendo gran ruido montado en su caballo negro. Habría sido el Apu Chamilco de la parte baja.

De regreso a la pareja Apu Doctor Manuel Ayavi y María Piti y Angas, la señora Juana asegura que María Piti y Angas hace daño o enferma a la gente. En una ocasión, don Manuel Salcedo, un agricultor y pastor de avanzada edad, estaba con sus 10 ovejas en la zona del Apu María Piti y Angas. Él solía tejer e hilar pues antaño los hombres hilaban para la bayeta y era un trabajo cotidiano.

Cuando llegó al sitio de María Piti, en medio de la neblina encontró cuatro naranjas en una piedra plana (maray) y asegura que ese lugar era su mesa. Se sentó al lado chacchando (masticando) su coca y solicitó al apu que lo ayude a cuidar sus ovejas y le dijo también que tenía deseos de comer sus naranjas, aunque con miedo. Al final, suponiendo que ya había percibido (comió) el olor de la fruta, se comió las cuatro naranjas, dejando algo de coca en su lugar como acto de reciprocidad con los apus. Mientras tanto, la neblina se disipó y encontró otras cuatro ovejas más, sumando a su manada; dudó pensando que podrían tener dueño y así los volvió a llevar a la zona esperando que apareciera el dueño, pero nadie apareció ni hubo reclamos. En conclusión, Apu María Piti escuchó su pedido y le entregó esas ovejas y desde entonces su ganado y sus animales se multiplicaron en beneficio de él y su familia. Al final de la vida de don Manuel Salcedo, los entendidos (pongos-curanderos) aseguraron que efectivamente el Apu María Piti le regaló aquellas ovejas y ahora ella acudía a don Manuel para recogerlo con todo su ganado. Según su relato, ella habría dicho: «Recogeré mis ganados con todo su dueño». Así todo terminó.

\section{Cambios climáticos, tensiones y desencuentros desde sus actores}

Al respecto de los trastornos climáticos, doña Juana cuenta con pesar que antes las familias atendían, cuidaban y pagaban a sus apus locales, pero que hoy eso ha cambiado. Cuidar de 
los apus era una situación fundamental para mantener el equilibrio con la naturaleza activa. Pero, en la actualidad, en la comunidad de Uripa y en otras zonas del distrito encontramos a familias que han abandonado el culto a los apus así como el catolicismo para abrazar otra religión (protestante o evangélica). Ella misma nos explica que hoy todo ha cambiado mucho. Las nuevas generaciones ya no toman en consideración a los apus, mientras que otros dicen que son cosas del diablo como los «evangélicos», quienes han proliferado en todas partes, negando a los dioses tutelares y acusándolos de ser «nuestros ídolos», al tiempo que rechazan las imágenes de los santos. Todo esto genera desconcierto e incertidumbre, no solo en la relación entre humanos, sino entre los humanos y el ecosistema, pensado este como activo, dinámico y cargado de fuerzas telúricas.

Todo esto tiene implicancias en la dinámica de la naturaleza y doña Juana lo sabe. Por eso, ella asegura que vivimos en «otro tiempo cambiado». Antes había mucha lluvia y ahora es escasa, es difícil planificar y los sembríos resienten esto. Cada vez más intervienen productos químicos en la producción, hay muchos desechos plásticos que se arrojan por todas partes, especialmente en los ríos, y eso es una forma de romper con el respeto y el cuidado recíproco entre seres humanos y naturaleza. Ella asegura que cuando fue niña no vio nada de esto. Se ha llegado a tal extremo que incluso a los niños no les gusta la comida del campo y prefieren los dulces, golosinas: «ya no quieren la cancha ni el mote».

Cree que el tiempo ha cambiado mucho, debido al maltrato de la tierra, haciendo referencia a los productos agroquímicos utilizados para los cultivos, y a la mucha suciedad que llega finalmente al mar. Ya no hay lluvia, solo sequía, granizada y mucha helada que afecta, provocando una baja en la producción. La mirada de la señora Juana es holística y relacionada con todos los elementos del ecosistema. Asegura que hay mucho calor, mucho viento y poca lluvia. Y ahora hay muchas enfermedades que atacan a las plantas y hasta a las flores del campo. La contaminación se propaga cuando lavan y arreglan los carros, hacen caer su aceite y este es llevado por la lluvia, contaminando los ríos. Todo eso era muy diferente, no solo eso que llamamos cambio climático genera estragos, sino también la vida moderna poco amable y reciproca con el medio ambiente.

Desde su perspectiva, no logra comprender el porqué de la ausencia o el retraso de las lluvias. Antaño, la dinámica de la lluvia seguía un ritmo normal y se podría prever la buena lluvia para el mes de agosto, entre los días 15 y 20. Pero ahora esto es incierto. El año pasado hubo algo más de lluvia, hoy esto ha cambiado. Lo que se siembra ya no produce bien y no sabe qué sucederá más adelante. Esto expresa el sentimiento generalizado sobre la incertidumbre que vive gran parte de la población en los Andes. Y lamenta que este sea el presente que heredarán los niños y las generaciones venideras.

En su experiencia, cuando hace mucho calor, es muy posible que caiga la granizada, por ello se arroja un cohete al cielo. Pero, en términos generales, las poblaciones andinas sugieren que la granizada tiene miedo a los fuegos artificiales; por esa razón, su uso es generalizado y todos los pobladores colaboran para su compra. Incluso, las familias creen que la granizada es ciega, pues no tiene dirección y es discontinua.

En los tiempos de su padre, cuando las nubes negras se arremolinaban en la dirección de su pueblo o sector, su padre disponía rápidamente de ajos, hierbas secas y buscaban los restos de la culebra (serpiente) y «guano» (estiércol). Todo se colocaba junto y se quemaba. Además, agarraban al chancho de las orejas, lo hacían bailar y el chancho hacía gran ruido, justamente, sus gritos y chillidos ahuyentaban el granizo. Y ahora ya nada de eso es posible. 
Finalmente, Juana recuerda que en una ocasión emprendió un viaje a Cocharcas con su padre. Ahí vieron que los mayores reunían a los niños en la iglesia y colocaban a la virgen en la puerta de la iglesia con el propósito de que todos los niños reunidos griten pidiendo que haga llover: «iTu lluvia suéltenos!», gritaban todos con gran furor. Tras estas rogativas, la tan ansiada lluvia llegaba a la región. Esa era la forma de pedir la lluvia en los tiempos antiguos, y ahora ya nadie lo recuerda.

\section{Discusión}

La evidencia etnográfica da cuenta de la experiencia de muchos campesinos, reflejada en la voz de la señora Juana de Uripa. Su experiencia es una ventana al mundo de los apus y su estrecha relación con los seres humanos.

Con respecto al cambio climático, que es centro de atención de este artículo, debemos señalar que la reflexión de la señora Juana es muy similar al de otros pobladores de los Andes. De hecho, siguiendo a Rudiak-Gould (2011), creemos que es sustancial penetrar en la percepción de las personas y las comunidades sobre el cambio climático, y en esa dirección, las y los campesinos coinciden al hacer referencia al actual desconcierto que causa la dinámica de las lluvias, la presencia de lluvias excesivas (lluvia loca) y el exceso de calor, lo que altera las dinámicas productivas y, por último, la vida, asumiéndolos como un proceso holístico e integral.

Y, en ese sentido, hemos tratado de recoger la visión y la experiencia de las familias en el campo. No obstante, como sugiere Norgaard (2011), es muy importante no solo apoyarnos en la evidencia de la experiencia local y de sus percepciones, sino también emprender la tarea de constituir un ejercicio interdisciplinario para interactuar a través de un análisis multinivel y modelizar los datos sobre el ecosistema, lo cual nos permitirá leer más a fondo la relación causal del cambio climático con la vida en el campo.

El testimonio de la señora Juana es al mismo tiempo el testimonio de muchas familias. Y a pesar que ellos están indicándonos una serie de señales muy claras sobre los impactos del cambio climático sobre la vida en el campo, se observa también la ausencia de acciones concretas de los gobiernos locales para enfrentar estos cambios. Si bien Rudiak-Gould (2011) asegura que la antropología muy frecuentemente se aboca a recoger la visión local y la experiencia concreta de las familias, también es clave repensar el discurso científico global sobre el cambio climático, el cual enfatiza mucho las medidas macro de adaptación. Sin embargo, es necesario también enfatizar el papel de las agendas locales para adaptarnos y reducir al máximo la vulnerabilidad, y con ello convertir esto en una posible oportunidad. Es interesante notar no solo el discurso científico, sino también el discurso gubernamental. Por ejemplo, en algunas zonas que fueron parte del trabajo de campo, obtuvimos datos que hacían referencia a la producción de palta Hass (Persea americana 'Hass) por encima de los 3000 m.s.n.m. Como bien señala Marino (2015) y Orlove et al. (2019), necesitamos retomar la dimensión política de este proceso, por lo que estamos convencidos de que es necesario pensar y actuar desde abajo, de la mano con las comunidades campesinas.

Muchos de los relatos recogidos y entrevistas realizadas en campo enfatizan el papel de las fuerzas tutelares como apoyo para mantener el equilibrio, aunque esto es una lucha constante, y no un proceso lineal, sino uno activo y dinámico. Al preparar la tierra y organizar el campo, las semillas son bendecidas en nombre de los apus locales. Ellos son en cierta forma la guía y el sustento, y todos hacen una parte de la tarea para ser parte de este equilibrio frágil. En ese sentido, concordamos con Graybill (2013), quien sugiere que existen aspectos emocionales ligados a la manera cómo entendemos y conceptualizamos el ecosistema y los problemas que acarrea aquello que llamamos cambio climático. De hecho, el testimonio de la señora Juana, así como 
de otros, manifiestan angustia, incertidumbre, pesar y autocrítica. Bien lo dice la señora Juana, el problema no es el futuro, el problema es el presente que estamos compartiendo con los niños. Por esa razón, es clave cuando Carothers et al. (2014) enfatizan la importancia de pensar el medio ambiente y el cambio climático bajo la perspectiva holística del mundo humano-ambiental, idea que también enfatizan Barnes et al. (2013) sobre el papel de la antropología para dar cuenta de la visión holística que conforman los sistemas humanos y naturales, así como del papel de los cambios (culturales, sociales, políticos y económicos).

El tema de la adaptación y la vulnerabilidad es uno que incluso podría ser revisado desde una aproximación antropológica para retener no solo el sentido de la experiencia social, sino también aspectos como los valores y la ética que están estrechamente vinculados, según sugiere Walshe y Argumedo (2016). Siguiendo ese hilo, coincidimos con Brugger y Crimmins (2013), quienes, aunque exploran comunidades rurales en el sudoeste de los Estados Unidos, aseguran que no debemos dejar de lado la manera tan singular de conceptualizar no solo el medio ambiente, sino también el tiempo como experiencia social; pero más importante aún, la discusión sobre el cambio climático que no debería de desvincularse de otros debates como la desigualdad, la justicia y el cambio social, así como también la necesidad de recuperar las tecnologías y formas de pensar del mundo andino vinculadas con el sistema ecológico. De hecho, no atender los efectos del cambio climático en los Andes coloca a estas poblaciones en una situación más difícil aún, a lo cual se suma las asimetrías, la pobreza y el arrinconamiento estructural. Finalmente, coincidimos con Roncoli et al. (2016) en el hecho de que la discusión sobre el cambio climático es algo que podría proyectarnos a problematizar y planificar acciones futuras concretas, que permitan superar actuales asimetrías, injusticias e inequidades.

En estos tiempos turbulentos del cambio climático, de conflictos sociales, ambientales y contaminación en todo el territorio, el papel de las montañas sagradas se hace más relevante para las familias en el campo. Las montañas sagradas como los apus y la pachamama son fuente para la inspiración y apoyo de cientos de familias, quienes confían en los valores que proyectan, al tiempo que actúan como un soporte simbólico y emocional en el marco de los sistemas rituales en los Andes. Ante la ausencia de lluvias, las familias recurren a sus apus locales y sus lagunas sagradas para rogar por buenas lluvias, abundante cosecha y bienestar para sus familias. Las diversas ofrendas y el alimento a los dioses, no solo va dirigido a las grandes montañas sagradas, sino incluye especialmente a los apus locales y a la pachamama.

En cada provincia visitada, existen montañas sagradas que se ubican en la parte más alta de la jerarquía simbólica, pero también existen montañas sagradas locales que se articulan con las montañas principales, formando una red simbólica, con atributos y cualidades. Hoy, las familias en los Andes, en medio de un contexto de conflictos, como la minería informal y otras formas de explotación de recursos naturales, en varios casos, se han convertido en guardianes celosos de sus apus y de sus lagunas, como en el caso del Apu Pisti de Aymaraes, solo para citar un ejemplo en el curso del trabajo de campo. Todo esto indica la centralidad que aun juegan los apus y la pachamama en los sistemas simbólicos, rituales y el diálogo sobre procesos globales como el cambio climático.

\section{Conclusiones}

El cambio climático constituye uno de los mayores desafíos globales, y esto supone el despliegue de un conjunto de actores a nivel global, como políticos, científicos, técnicos y programas de cooperación. Sin embargo, también debemos reconocer que la manera cómo experimentamos el cambio climático no es homogéneo a lo largo de las comunidades asentadas en la cadena de 
montañas de los Andes. Las poblaciones, si bien es cierto enfrentan las mismas variaciones, no responden de la misma manera. Hay variaciones en las visiones y experiencias locales sobre este proceso global. Y en esa dirección, las variables de género, generacionales y socio-económicas, e incluso demográficas, son también claves y deben ser reintroducidas en análisis posteriores. No solo se trata de captar los sentidos y significados del cambio climático, sino también de generar un conocimiento situado y enriquecido por una aproximación antropológica y holística, evitando todo riesgo de esencialismo.

Finalmente, creemos que no solo debemos informar sobre la experiencia local, sino advertir sobre la necesidad de animar agendas locales desde abajo, para pensar y actuar ante el cambio climático, sin soslayar las profundas desigualdades y asimetrías presentes en la larga duración de las poblaciones andinas. Necesitamos poner lo mejor de nuestro arte y ciencia para pensar de manera colaborativa el impacto del cambio climático, e imaginar nuevas formas para afrontar estos desafíos.

\section{Bibliografía}

Andía, J. J. R. (2003). La fiesta del ganado en el valle de Chancay, 1962-2002: ritual, religión y ganadería en los Andes: etnografía, documentos inéditos e interpretación. Lima: Fondo Editorial de la Pontifica Universidad Católica del Perú.

Arroyo, S. (2006). Culto a los Hermanos Cristo. Sistema religioso andino y cristiano: Redes y formas culturales del poder en los Andes (tesis doctoral). Universidad Nacional Mayor de San Marcos, Lima.

Arroyo, S. (2012). De santiago mataindios a Tayta Shanti. Investigaciones sociales, 16(29), 159-169.

Balter, M. (2007). Seeking agriculture's ancient roots. Science, 316(5833), 1830-1835.

Barnes, J., Dove, M., Lahsen, M., Mathews, A., McElwee, P., McIntosh, R., ... \& Yager, K. (2013). Contribution of anthropology to the study of climate change. Nature Climate Change, 3(6), 541-544.

Bogin, B. (1982). Climate change and human behavior on the southwest coast of Ecuador. Central Issues in Anthropology, 4(1), 21-31.

Brosseder, C. (2014). The power of huacas: change and resistance in the Andean world of colonial Peru. Austin: University of Texas Press.

Brugger, J., y Crimmins, M. (2013). The art of adaptation: Living with climate change in the rural American Southwest. Global Environmental Change, 23(6), 1830-1840.

Bugallo, A. (2014). Ontología relacional y ecosofía en Arne Naess. Nuevo Pensamiento, 1(1), 151-174.

Carothers, C.; Brown, C.; Moerlein, K. J.; López, J. A.; Andersen, D. B. y Retherford, B. (2014). Measuring perceptions of climate change in northern Alaska: pairing ethnography with cultural consensus analysis. Ecology and Society, 19(4). https://bit.ly/3oe6zQi

Crumley, C. L. (2018). Historical ecology. The International Encyclopedia of Anthropology. Oxford: Wiley.

Ceruti, M. C. (2013). Chachani, Misti y Pichu Picchu: pasado y presente de los volcanes sagrados de Arequipa. Anuario de Arqueología, Rosario, 5(1):359-372.

Cometti, G. (2020). A Cosmopolitical Ethnography of a Changing Climate among the Q'ero of the Peruvian Andes. Anthropos, 115(1), 37-52.

De Menocal, P B. 2001. Cultural responses to climate change during the late holocene. Science, (292), 667-672.

Eliade, M (1981). Lo sagrado y lo profano, volumen 3. Barcelona: Labor.

Estermann, J. (2013). Ecosofía andina: Un paradigma alternativo de convivencia cósmica y de Vivir Bien. Revista FAIA, 2(9), 2-21.

Fuenzalida, F. (1979). El Cristo pagano de los Andes: una cuestión de identidad y otra sobre las eras solares. Debates en Sociología, (4), 1-10. 
Fuenzalida, F. (1980). Santiago y el Wamani: Aspectos de un culto pagano en Moya. Debates en Sociología, (5), 155-187.

Golte, J. (2003). La construcción de la naturaleza en el mundo prehispánico andino, su continuación en el mundo colonial y en la época moderna. Revista de Antropología, 3(3), 13-59.

Golte, J. (2004). Divinidades femeninas moche. En Dorothea Ortmann (Comp.). Anuario de ciencias de la religión: las religiones en el Perú de hoy (pp. 165-220). Lima: Fondo Editorial de la Universidad Nacional Mayor de San Marcos y Consejo Nacional de Ciencia, Tecnología e Innovación Tecnológica.

Graybill, J. K. (2013). Imagining resilience: situating perceptions and emotions about climate change on Kamchatka, Russia. GeoJournal, 78(5), 817-832.

Guamán Poma de Ayala, F. (1980). Nueva corónica y buen gobierno, volumen 2. Caracas: Fundacion Biblioteca Ayacucho.

Guzman, M. (2019). Arquitectura y paisaje simbólico en los andes centrales. Arquitextos, (31), 11-30.

Juárez, M. G. (2015). Arquitectura Chancay. El tinkuy en la organización espacial. Arqueología y Sociedad, (30), $9-25$.

Jurt, C.; Burga, M. D.; Vicuña, L.; Huggel, C. y Orlove, B. (2015). Local perceptions in climate change debates: insights from case studies in the Alps and the Andes. Climatic Change, 133(3), 511-523.

Kuper, R., \& Kröpelin, S. (2006). Climate-controlled Holocene occupation in the Sahara: motor of Africa's evolution. Science, 313(5788), 803-807.

Lecaros-Terry, A. T. (2001). Los peregrinos del Señor de Muruhuay: espacio, culto e identidad en los Andes (tesis doctoral). Freie Universität, Berlin.

Maffesoli, M. (2017). Ecosofia: sabedoria da casa comum. Revista FAMECOS: mídia, cultura e tecnologia, 24(1). https://bit.ly/2SNfGfb

Marino, E. (2015). Fierce climate, sacred ground: An ethnography of climate change in Shishmaref, Alaska. Fairbanks: University of Alaska Press.

Martínez Rica, J. P. (2003). Ecología de montaña. Revista Ibón, (30), 8-15.

McIntosh, R. J. (2000). Social memory in Mande. The way the wind blows: climate, history, and human action. Nueva York: Columbia University Press.

Migowski, C., Stein, M., Prasad, S., Negendank, J. F., \& Agnon, A. (2006). Holocene climate variability and cultural evolution in the Near East from the Dead Sea sedimentary record. Quaternary Research, 66(3), 421-431.

Millones, L. (2011). El encuentro o tinkuy en textos coloniales andinos. https://bit.ly/3bnv8Fo

Mujica, L. (2017). Pachamama kawsan: hacia una ecología andina. Lima: Pontificia Universidad Católica del Perú, Instituto de Ciencias de la Naturaleza, Territorio y Energías Renovables.

Norgaard, K. M. (2011). Living in denial: Climate change, emotions, and everyday life. Cambridge: MIT Press.

Oldfield, F. (1993). Forward to the past: changing approaches to Quaternary palaeoecology. En: Climate change and human impact on the landscape (pp. 13-21). Dordrecht: Springer.

Orlove, B.; Milch, K.; Zaval, L.; Ungemach, C.; Brugger, J.; Dunbar, K.; y Jurt, C. (2019). Framing climate change in frontline communities: anthropological insights on how mountain dwellers in the USA, Peru, and Italy adapt to glacier retreat. Regional Environmental Change, 19(5), 1295-1309.

Paerregaard, K. (2013). Bare rocks and fallen angels: Environmental change, climate perceptions and ritual practice in the Peruvian Andes. Religions, 4(2), 290-305.

Paerregaard, K. (2020). Communicating the Inevitable: Climate Awareness, Climate Discord, and Climate Research in Peru's Highland Communities. Environmental Communication, 14(1), 112-125.

Peterson, L. C., \& Haug, G. H. (2005). Climate and the collapse of Maya civilization: A series of multi-year droughts helped to doom an ancient culture. American Scientist, 93(4), 322-329.

Polia Meconi, M. (1996). Despierta, remedio, cuenta...: adivinos y médicos del Ande. Lima: Fondo Editorial de la Pontifica Universidad Católica del Perú.. 
Reinhard, J. (2012). Las montañas Sagradas y las culturas preincaicas de los Andes. En Margarita Chávez y Peniche, Stanislaw Iwaniszewski y Ricardo Cabrera. América: tierras de montañas y volcanes. Huellas de la Arqueología (pp. 51-71). México D.F.: Instituto Nacional de Antropología e Historia.

Ricard Lanata, X. (2007). Ladrones de sombra. El universo religioso de los pastores del Ausangate. Lima: Instituto Francés de Estudios Andinos y Centro Bartolomé de Las Casas.

Richerson, P. J., Boyd, R., \& Bettinger, R. L. (2001). Was agriculture impossible during the Pleistocene but mandatory during the Holocene? A climate change hypothesis. American Antiquity, 66(3), 387-411.

Ríos, M. A. (2004). El Maestro Curandero en el Perú: Antecedentes históricos de su aparición y su importancia en el siglo XXI. Sociedad y discurso, (6), 1-30.

Roncoli, C.; Crane, T., y Orlove, B. (2016). Fielding climate change in cultural anthropology. En Anthropology and climate change (pp. 87-115). Londres: Routledge.

Rudiak-Gould, P. (2011). Climate change and anthropology: The importance of reception studies. Anthropology Today, 27(2), 9-12.

Sánchez Garrafa, R. (2006). Apus de los Cuatro Suyos: construcción del Mundo en los Ciclos Mitológicos de las Deidades Montaña. Lima: Instituto de Estudios Peruanos.

Sapkota, P.; Keenan, R. J.; Paschen, J. A.; y Ojha, H. R. (2016). Social production of vulnerability to climate change in the rural middle hills of Nepal. Journal of Rural Studies, 48, 53-64.

Schlegelberger, B. (1996). Aspectos cosmológicos de la religiosidad andina. En Max Peter Baumann. Cosmología y música en los Andes (pp. 271-282). Madrid: Iberoamericana.

Vitry, C. (2002). Apachetas y mojones, marcadores espaciales del paisaje prehispánico. Revista Escuela de Historia, 1(1), 179-191.

Vitry, C. (2005). Contribución al estudio de caminos de sitios arqueológicos de altura. Volcán Llullaillaco (6739 m). Salta-Argentina. En A. Austral y M. Tamagnini. Problemáticas de la Arqueología Contemporánea. Actas PostCongreso del XV Congreso Nacional de Arqueología Argentina (pp. 127-140). Río Cuarto: Editorial de la Universidad Nacional de Río Cuarto,.

Vitry, C. (2017). El rol del Qhapaq Ñan y los Apus en la expansión del Tawantinsuyu. Boletín del Museo Chileno de Arte Precolombino, 22(1), 35-49.

Walshe, R. y Argumedo, A. (2016). Ayni, Ayllu, Yanantin and Chanincha: the cultural values enabling adaptation to climate change in communities of the potato park, in the Peruvian Andes. GAIA-Ecological Perspectives for Science and Society, 25(3), 166-173.

Weber-Alvarez, A. (2018). Global climate change is highly local: Perception and adaptation of the international climate change discourse in Peru-a case study of the Southern Peruvian Andes (tesis doctoral). Fakultät für Angewandte Informatik Institut für Geographie der Universität Augsburg, Augsburgo.

Vallejo, R. y Alvarado, S. (2015). El eterno retorno del fenómeno de las heladas en el Perú: ¿Existen adecuadas políticas para combatir dicho fenómeno en el Perú? https://bit.ly/3y85BcT

Presentado: 31/08/2020

Aceptado: 28/05/2021

Publicado online: 10/08/2021 